\title{
Chronic myeloid leukaemia with lymphosarcoma
}

\author{
J. S. HOWELL AND A. G. W. WHITFIELD
}

From the Department of Pathology, University of Birmingham, and the United Birmingham Hospitals

SYNOPSIS The clinical and pathological features of two patients with chronic myeloid leukaemia who died from lymphosarcoma are described. Both had been treated with busulphan which, at the time of development of lymphosarcoma and at death, appeared to be controlling the leukaemia satisfactorily.

The origin of myeloid cells from lymphocytes has been suggested by Maximow and Bloom (1948) and this concept has been supported by Yoffey, Thomas, Moffatt, Sutherland, and Rosse (1961), but Ham (1961) casts considerable doubt on the hypothesis. In this controversy, observations which show that a neoplastic process involving myeloid cells can be converted into, or coexist with, a neoplastic process involving lymphoid cells are of considerable interest. The two cases reported here show such a situation.

\section{CASE REPORTS}

CASE 1. J. W., aged 44 years, early in 1957 began to notice weakness, lassitude, and gradual loss of weight. In August 1958 he injured his shin and a large haematoma developed; this was associated with pyrexia up to $103 \cdot 5^{\circ} \mathrm{F}$.

Received for publication 26 February 1963 which gradually subsided over the next month. Shortlys after recovery from the leg injury he developed suddentransient bilateral blindness, and afterwards vision ress mained blurred. Ophthalmoscopic examination showedo bilateral papilloedema and retinal haemorrhages. The systemic blood pressure was normal. The liver was slightly enlarged and the spleen was readily palpable $\stackrel{\mathbb{D}}{\mathbb{C}}$ there was no lymphadenopathy. A blood count at this time showed: $\mathrm{Hb} 64 \%,(9 \cdot 4$ g. $\%$ ) W.B.C. $364,000 /$ c.mm. approximately $60 \%$ of which were premyelocytes and metamyelocytes and the rest mainly granulocytes. A few myeloblasts and occasional nucleated red cells were alsos present. The platelet count was $310,000 / \mathrm{c} . \mathrm{mm}$. Marrow films were not examined, but the peripheral blood picturen was typical of chronic myeloid leukaemia.

He was treated with busulphan, the dose varying be tween $2 \mathrm{mg}$. and $6 \mathrm{mg}$. daily according to the white celb count (Fig. 1). He remained well and white cell counts were in the region of $6,000 / \mathrm{c} . \mathrm{mm}$. with virtually norma

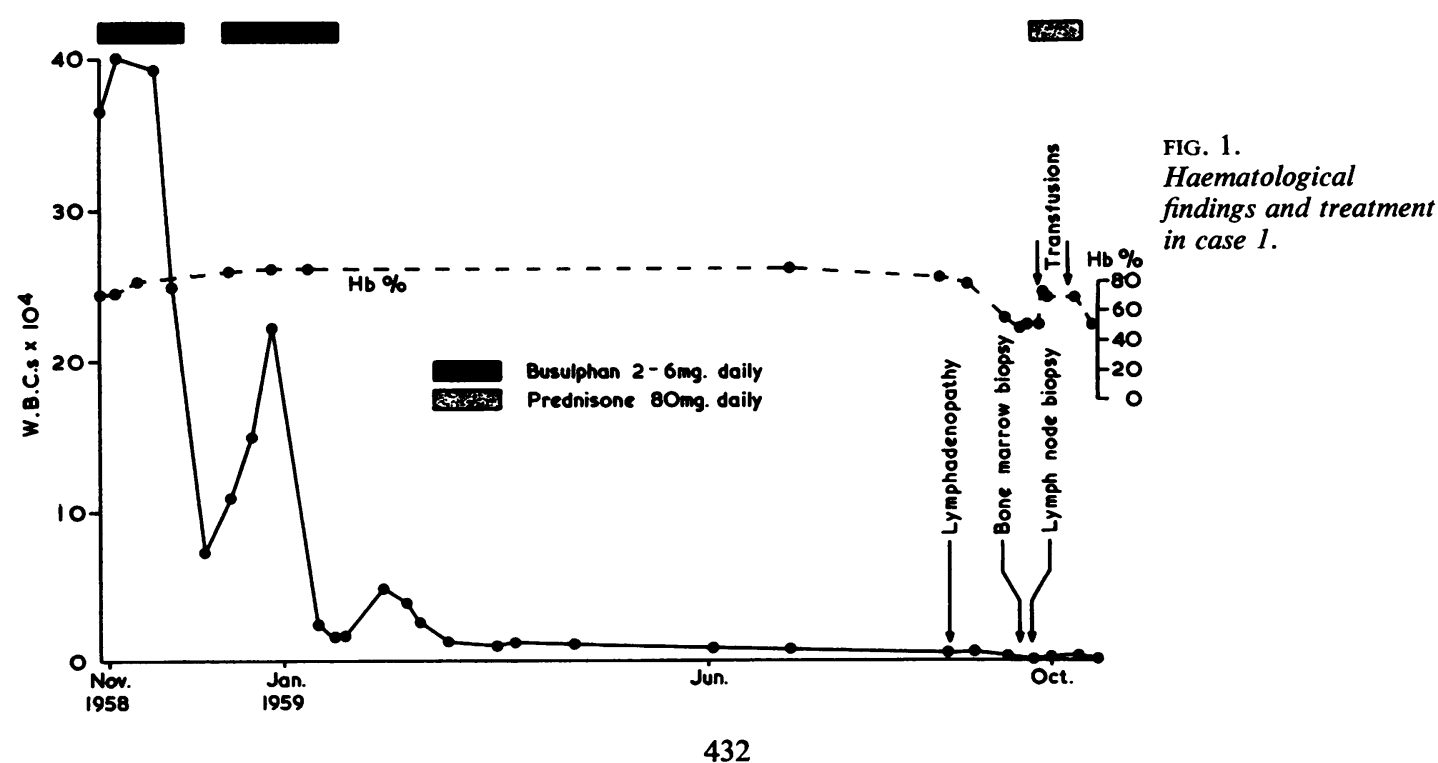




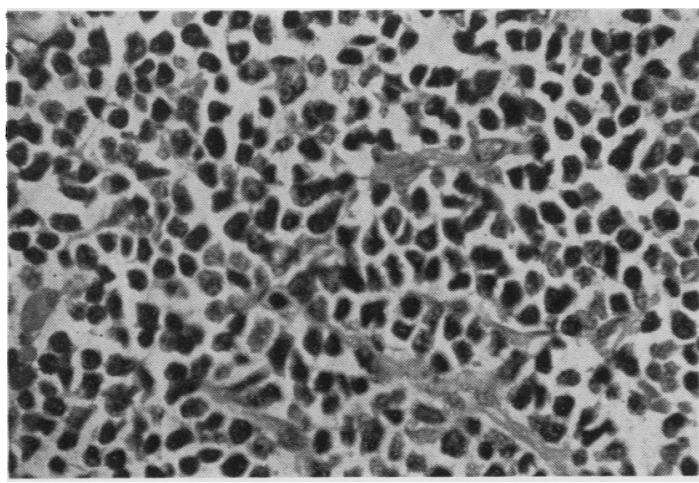

FIG. 2

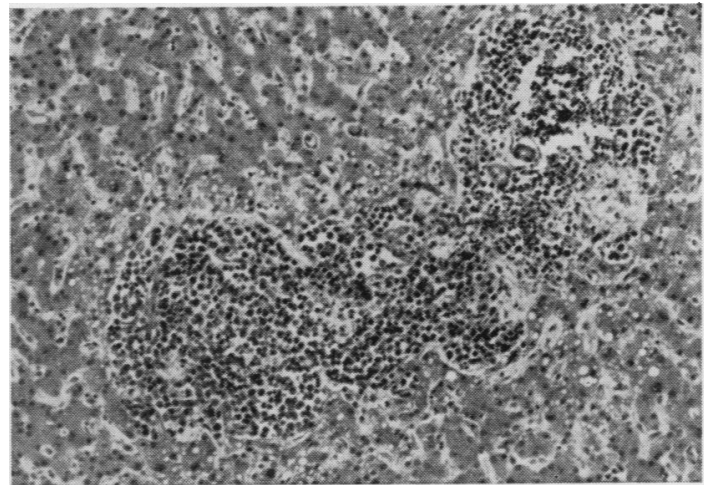

FIG. 3

FIG. 2. Lymph node biopsy showing lymphosarcoma. (Haematoxylin and eosin $\times 450$.)

FIG. 3. Expansion of portal tracts by cellular infiltrate. Note absence of infiltration of sinusoids. (Haematoxylin and eosin $\times$ 100.)

differentials until August 1959 when he developed a marked and generalized lymphadenopathy with firm lymph nodes up to 1 in. in diameter in the neck, axillae, and groins. The spleen was just palpable but the liver was not felt.

The total W.B.C. was $6,600 /$ c.mm. with a normal differential count and no primitive cells. Bone marrow smears were extremely cellular with areas showing very marked excess of myeloblasts, myelocytes, and metamyelocytes, the appearances being consistent with myeloid leukaemia without release of primitive cells into the blood.

A cervical lymph node was biopsied which microscopically (Fig. 2) showed the appearances of lymphosarcoma. The architecture of the node was completely destroyed and replaced by cells which were fairly uniform in size and appearance with scanty cytoplasm and large round, darkly staining, faintly vesicular nuclei with occasional nucleoli; mitotic figures were numerous. These cells extended through the capsule to the adjacent fibrofatty tissue and similar cells were also present in veins. There was no increase in reticulin fibre and the peroxidase reaction was negative.

He became increasingly anaemic and the white cell count fell to $1,800 / \mathrm{c} . \mathrm{mm}$. with thrombocytopenia. Prednisone, $80 \mathrm{mg}$. daily, and blood transfusions were given but his condition rapidly deteriorated and he developed a purpuric rash on the legs and ulcerative lesions in the mouth and pharynx. The purpura gradually became more widespread and death in coma occurred on 28 October 1959.

Necropsy The cervical, inguinal, axillary, thoracic, mesenteric, paraortic, and pelvic lymph nodes were enlarged, matted together, and presented fleshy, pale cut surfaces with areas of haemorrhagic necrosis. The liver $(2,700$ g.), spleen (1,300 g.), and kidneys (440 g.) were enlarged and all showed evidence of neoplastic infiltration. Red marrow had extended throughout the entire length of the femur.

Microscopically, the lymph nodes from the various sites examined showed appearances essentially similar to those seen in the biopsy specimen. In the liver there was infiltration with cells similar to those in the lymph nodes but these were entirely confined to the expanded portal tracts (Fig. 3). The normal architecture of the spleen was destroyed by similar cellular infiltration. Small cellular infiltrates were present in the cortex of both kidneys. Peroxidase reactions on all the tissues examined were negative.

CASE 2 G. H., aged 73 years, in July 1958 had a coronary thrombosis and was admitted to hospital where he was also found to have splenomegaly, and a blood count showed the typical features of chronic myeloid leukaemia. After recovery from the myocardial infarct he had angina of effort for which, four months later, he was referred to one of us. Abnormalities observed at this time were pallor, ankle oedema, and splenomegaly. An electrocardiogram showed a healed posterior myocardial infarct. His blood showed $\mathrm{Hb} 66 \%(9.6$ g. $\%)$ total W.B.C. $315,000 /$ c.mm. (37\% polymorphs, $20 \%$ myelocytes, $17 \%$ metamyelocytes, $5 \%$ premyelocytes), and platelets $312,000 /$ c.mm. (Fig 4). He was treated with demecolcine, $10 \mathrm{mg}$. daily, but after six weeks there was only relatively slight improvement in the blood picture, and therapy was changed to busulphan, $6 \mathrm{mg}$. daily. Response was slow but after three months the $\mathrm{Hb}$ level was $82 \%(12.0 \mathrm{~g} . \%)$ total W.B.C. $30,000 /$ c.mm., with only $6 \%$ primitive forms, and the spleen was impalpable. Though recurrent elevations of the white cell count necessitated two further courses of busulphan he remained well until September 1960 when pericarditis developed, two small nodules appeared in the skin over the right iliac fossa, and on rectal examination a tumour-like mass was felt which did not appear to be either rectal or prostatic in origin. The peripheral blood showed the leukaemia to be well controlled, there were no enlarged lymph nodes, and the spleen was only just palpable. His condition rapidly deteriorated and he died seven days later.

Necropsy The heart (630 g.) showed a fibrinous peri- 


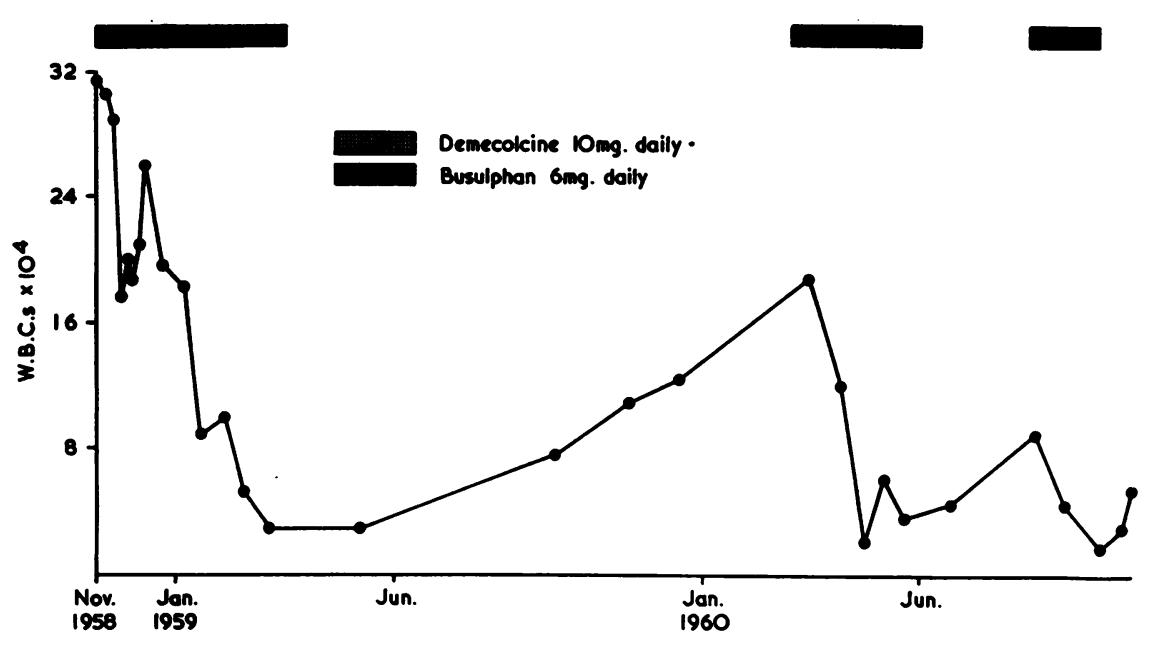

FIG. 4.

Haematological findings and treatment in case 2.

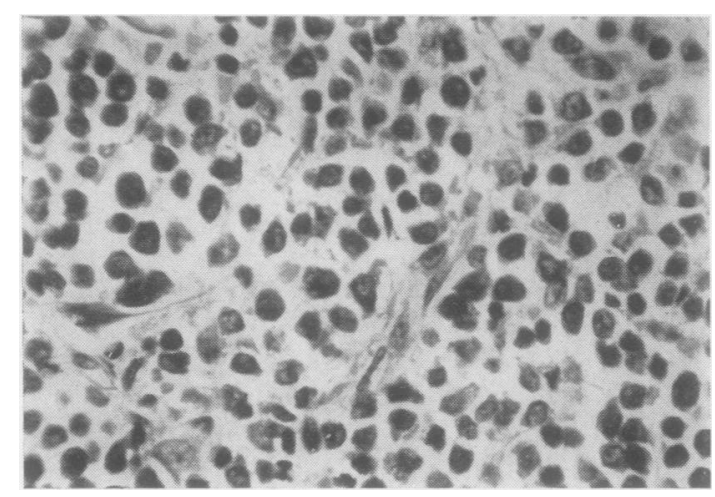

FIG. 5. Lymph nodes (at necropsy) showing lymphosarcoma. (Haematoxylin and eosin $\times 450$.)

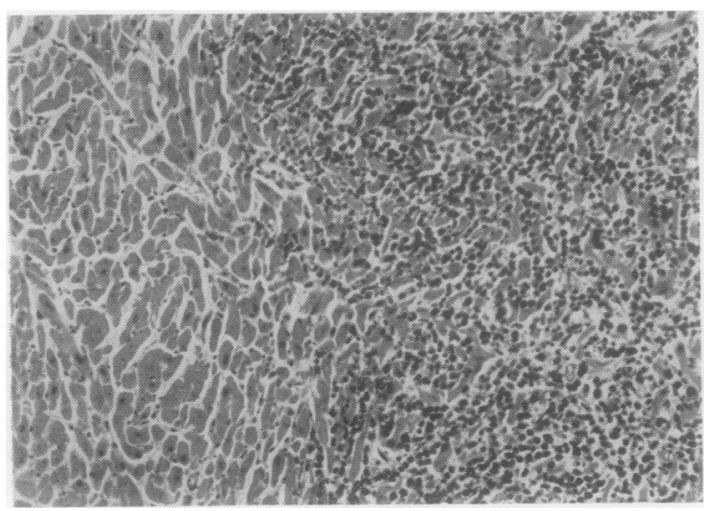

FIG. 6. Myocardium showing infiltration by lymphosarcoma. (Haematoxylin and eosin $\times 100$.) carditis and a firm mass of white tumour tissue was ad- $\frac{\mathbb{\mathbb { D }}}{2}$ herent to the anterior aspect of the parietal pericardium. $\frac{3}{8}$ Similar tumour tissue was found beneath the endocar- $\underline{\mathbb{D}}$ dium growing down into the right ventricular wall and in $\vec{\theta}$ places completely penetrating the muscle. The upper part $\circ$ of the left ventricle was also invaded by tumour which ${ }^{\omega}$ involved the adventitia of the aorta. In addition there was much fibrosis of the posterior wall of the left ventricle and the lumen of the coronary arteries was virtually obliterated by advanced atheroma.

The thoracic and abdominal lymph nodes were greatly $\frac{0}{\Phi}$ enlarged and replaced by white, fleshy, homogeneous $\varrho$ tumour tissue. Extensive deposits of tumour in the retro-홍 peritoneal tissues completely surrounded the kidneys and 3 adrenals. Further tumour deposits were found in the wall of the stomach forming polypoid projections into the lumen. The pancreas was almost completely replaced by tumour. The liver $(2,000 \mathrm{~g}$.) and spleen $(530 \mathrm{~g}$.) were? moderately enlarged.

Microscopic examination showed the normal architec- -3 . ture of the lymph nodes to be destroyed and replaced by cells which were relatively uniform in size with scanty cytoplasm and darkly staining vesicular nuclei; occasionalo eosinophilic nucleoli were present. The appearances were those of lymphosarcoma (Fig. 5). Similar cells wereo present in the subcutaneous nodules, in the polypoid masses of the stomach, and in the retroperitoneal mass. No In the heart there was considerable infiltration of the interstitial tissue causing separation and distortion of 0 individual muscle fibres (Fig. 6). The liver was virtually ${ }^{\omega}$ normal with only occasional microscopic foci of tumouro cells in the sinusoids. The spleen was congested ando showed some infiltration by primitive lymphoid cells. The kidneys also showed cellular infiltration but this was focal in distribution. Peroxidase reactions on the material ${ }^{+}$ examined were negative.

\section{DISCUSSION}

There are many reports in the literature of leukaemia $\stackrel{\frac{\pi}{2}}{\stackrel{2}{0}}$ 
complicating various forms of reticulosis, the best known example being chronic lymphatic leukaemia and lymphosarcoma. Cases of reticulum cell sarcoma terminating suddenly with acute leukaemia (Beutler, 1954; Zeffren and Ultmann, 1960) have been described. Leukaemia complicated by other forms of malignant disease, generally of epithelial origin, have also been observed (Moertel and Hagedorn, 1957).

However, the coexistence, or conversion, of myeloid leukaemia into a malignant reticulosis appears to be distinctly uncommon and we have been able to find only seven isolated case reports in the literature. Skworzoff (1930) described a child of 8 years who had myeloid leukaemia and developed a generalized lymphadenopathy due to Hodgkin's disease. Hanns, Israel, and Sacrez (1934) reported a 30-year-old man who had myeloid leukaemia treated by radiotherapy to the spleen with clinical improvement. Shortly afterwards a mass appeared in the lumbo-sacral region followed by other masses in soft tissues, which on biopsy were deposits of reticulum cell sarcoma. Wilcken (1957) described chronic myeloid leukaemia in a 25 -year-old woman treated by radiotherapy over the following three years with good remissions. During the final admission it was noted that she had enlarged lymph nodes in the axillae, lumps in both breasts, and pelvic masses. A lymph node biopsy showed reticulum cell sarcoma, which was confirmed at necropsy. Belolipetskaya and Gets (1960) described a similar case, a woman aged 22 who had myeloid leukaemia treated by cytotoxic drugs for three years, following which she developed reticulum cell sarcoma.

The association of myeloid leukaemia with lymphosarcoma was described by Yang (1936) in a young man who had chronic myeloid leukaemia treated by radiotherapy and one year later presented with generalized lymphadenopathy which on biopsy (from two sites) showed replacement of the nodes by lymphosarcoma. Forkner (1938) also very briefly described two cases of myeloid leukaemia coexisting with lymphosarcoma but one of these was probably the case described by Yang (1936) above. Sardesai and Bhatia (1959) described a 48-year-old man with generalized lymphadenopathy and enlargement of the liver and spleen. The peripheral blood contained 58,200 W.B.C.s/c.mm., including many premyelocytes and metamyelocytes. Bone marrow biopsy confirmed the diagnosis of myeloid leukaemia and an initial response to busulphan (myleran) was observed. However, a lymph node biopsy showed the typical appearances of lymphosarcoma.

It is difficult to account for the sequence of events in the two cases described here and no conclusions can be drawn regarding possible mechanisms whereby well-established chronic myeloid leukaemia is converted into or coexists with lymphosarcoma. However, it may be of some significance that these two cases, as well as similar cases abstracted from the literature, have been treated either with alkylating agents such as busulphan and/or radiotherapy. Radiotherapy and the alkylating agents are cytotoxic, mutagenic, and are themselves carcinogenic. It is possible that these agents while controlling myeloid proliferation allowed the stimulus initiating proliferation to be transferred in part to other cells, insusceptible to such control, thus altering the overt clinical and pathological manifestation of the disease.

We are greatly indebted to Dr. W. J. Walkden, Professor Melville Arnott, and Dr. J. Marshall Chalmers for allowing us to publish case 1, and to Dr. J. Marshall Chalmers for his constant help and guidance in the management of case 2.

\section{REFERENCES}

Belolipetskaya, T. A., and Gets, I. I. (1960). Arkh. Pat., 22 (3), 61. (Abstr. in Abstr. Soviet Med., 1961, 5, 241.)

Beutler, E. (1954). Ann. intern. Med., 40, 1217.

Forkner, C. E. (1938). Leukaemia and Allied Disorders, p. 127. Macmillan, New York.

Ham, A. W. (1961). Histology, 4th ed., p. 188. Pitman, London.

Hanns, A., Israel, L., and Sacrez, R. (1934). Strasbourg méd., 94, 42. (Abstrs. in Amer. J. Cancer., 1934, 21, 968.)

Maximow, A. H., and Bloom, W. (1948). Textbook of Histology, 5th ed., p. 107. Saunders, Philadelphia and London.

Moertel, C. G., and Hagedorn, A. B. (1957). Blood, 12, 788.

Sardesai, H. V., and Bhatia, K. C. (1959). Indian J.med. Sci., 13, 849.

Skworzoff, M. A. (1930). Frankfurt. Z. Path., 40, 81.

Wilcken, D. E. L. (1957). Med. J. Aust., 1, 204.

Yang, C. S. (1936). Chin. med. J., 50, 1153.

Yoffey, J. M., Thomas, D. B., Moffatt, D. J., Sutherland I. H., and Rosse, C. (1961). In Biological Activity of the Leucocyte, Ciba Foundation Study Group, No. 10, p. 45. Churchill, London.

Zeffren, J. L., and Ultmann, J. E. (1960). Blond, 15. 277. 